\title{
Article \\ Effects of Colonic Fermentation Products of Polydextrose, Lactitol and Xylitol on Intestinal Barrier Repair In Vitro
}

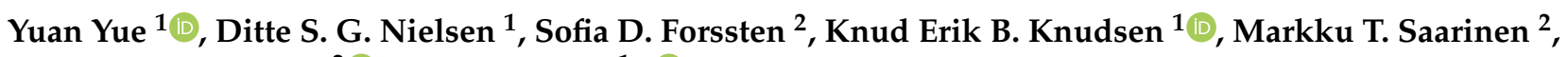 \\ Arthur C. Ouwehand ${ }^{2}{ }^{-1}$ and Stig Purup ${ }^{1, * \mathbb{C}}$ \\ 1 Department of Animal Science, Aarhus University, Foulum, Blichers Allé 20, DK-8830 Tjele, Denmark; \\ yuan.yue@anis.au.dk (Y.Y.); dittesgn@hotmail.com (D.S.G.N.); knuderik.bachknudsen@anis.au.dk (K.E.B.K.) \\ 2 DuPont Nutrition \& Biosciences, Global Health and Nutrition Science, Sokeritehtaantie 20, 02460 Kantvik, \\ Finland; Sofia.Forssten@dupont.com (S.D.F.); Markku.Saarinen@dupont.com (M.T.S.); \\ Arthur.Ouwehand@dupont.com (A.C.O.) \\ * Correspondence: Stig.purup@anis.au.dk
}

check for

updates

Citation: Yue, Y.; Nielsen, D.S.G.;

Forssten, S.D.; Knudsen, K.E.B.;

Saarinen, M.T.; Ouwehand, A.C.;

Purup, S. Effects of Colonic

Fermentation Products of

Polydextrose, Lactitol and Xylitol on Intestinal Barrier Repair In Vitro.

Appl. Sci. 2021, 11, 4174.

https://doi.org/10.3390/app11094174

Academic Editor: Hae-Jeung Lee

Received: 30 March 2021

Accepted: 1 May 2021

Published: 3 May 2021

Publisher's Note: MDPI stays neutral with regard to jurisdictional claims in published maps and institutional affiliations.

Copyright: (c) 2021 by the authors. Licensee MDPI, Basel, Switzerland. This article is an open access article distributed under the terms and conditions of the Creative Commons Attribution (CC BY) license (https:/ / creativecommons.org/licenses/by/ $4.0 /)$.

\begin{abstract}
Many functional food ingredients improve intestinal barrier function through their colonic fermentation products short chain fatty acids (SCFAs). Effects of individual SCFAs have been well studied, but the effects of SCFA mixtures-colonic fermentation products have been rarely investigated. Therefore, this study used an EnteroMix semi-continuous model to simulate the colonic fermentation of three widely used food ingredients, polydextrose, lactitol and xylitol in vitro, and investigated the effects of their fermentation products on impaired colonic epithelial barrier function through a mucus-secreting human HT29-MTX-E12 cell model. Fermentation of polydextrose and lactitol produced mainly acetate, while fermentation of xylitol produced mainly butyrate and resulted in a much higher butyrate proportion. All fermentation products significantly improved intestinal barrier repairing as measured by increased transepithelial electrical resistance and decreased paracellular permeability. Among these, xylitol fermentation products exhibited better repairing effects than that of polydextrose and lactitol. Correlation analysis showed that the repairing effects were attribute to butyrate but not acetate or propionate, implying that in the fermentation products butyrate may play a major role in improving intestinal barrier function. Our results suggest that functional food ingredients that mainly produce butyrate during fermentation may be of more value for improving gut health related to chronic diseases.
\end{abstract}

Keywords: short-chain fatty acids (SCFAs); in vitro colonic fermentation; intestinal barrier function; lactitol; polydextrose; xylitol

\section{Introduction}

The intestinal barrier is composed of gut microbiota, mucus, epithelial cells and some immune cells [1]. It is an important physical barrier that protects the underlying tissue from various pathogens and toxic substances [1]. Impaired intestinal barrier function has been associated with many chronic gastrointestinal diseases like coeliac disease, colorectal cancer, inflammatory bowel diseases, and also contributes to systemic diseases such as diabetes, allergic diseases and even chronic kidney disease [1-3]. Therefore, the maintenance of a healthy intestinal barrier, especially the repair of impaired barrier, is of great importance for the improvement of chronic diseases.

Several studies have highlighted the role of short chain fatty acids (SCFAs) in such areas. They are known to serve as energy source for colonocytes, enhance intestinal barrier function, and help to repair wounded epithelium [4-6]. The three major SCFAs, acetate, propionate and butyrate, have demonstrated positive effects on intestinal barrier function in vitro [7,8], ex vivo [9], and in vivo [10-12]. However, the level of individual SCFA required for improving intestinal barrier function seems to be different $[7,8,13]$. Moreover, 
SCFAs are naturally produced in the colon by microbial fermentation of undigested carbohydrates, but the composition of SCFAs in the fermentation products differs depending on the source, microbiota composition, site of fermentation, and host genotype [14,15]. Thus, SCFA composition in different colonic fermentation products can vary significantly, which may affect intestinal barrier function differently. Unfortunately, there are few studies assessing the effects of mixed SCFAs or colonic fermentation products on intestinal barrier function.

Polydextrose (PDX), lactitol and xylitol are ingredients widely used in THE food industry. They are non-digestible carbohydrates that can be fermented by colonic microbiota and have shown prebiotic properties [16-18]. The SCFA composition of fermentation products has been analyzed previously $[19,20]$; however, the effects of these fermentation products on intestinal barrier function have not been evaluated. The EnteroMix in vitro model is a semi-continuous fermentation simulator that is used for studying colonic microbial fermentation, and has shown good correlations with in vivo studies [21]. Through four sequentially connected vessels that simulate ascending, transverse, descending and sigmoid colon, the EnteroMix model is able to achieve semi-continuous influx of nutrients and efflux of waste products, thereby providing a physicochemical condition that closely resembles the in vivo environment [21]. The HT29-MTX-E12 (E12) cell line is a subclone of human colorectal adenocarcinoma cell line HT29-MTX [22]. Because of its characterization on tight junction formation and adherent mucus production [22], E12 cells are highly suitable for studying intestinal barrier function. In the current study, the EnteroMix model and E12 cell model were combined. SCFA production following in vitro fermentation of PDX, lactitol and xylitol using EnteroMix model was analyzed, and the effects of the fermentation products on impaired colonic epithelial barrier function was investigated by an established HT29-MTX-E12 cell model [23]. The purpose of the study was to investigate the effects of the fermentation products of two widely used sweeteners, lactitol and xylitol, and one prebiotic, polydextrose, on impaired colonic epithelial barrier function. It was hypothesized that fermentation products of PDX, lactitol and xylitol have different beneficial effects on the repair of impaired intestinal epithelial barrier, and this discrepancy is due to different SCFA production.

\section{Materials and Methods}

\subsection{In Vitro Fermentations}

The in vitro fermentation was performed using the EnteroMix model as previously described [24,25]. Each simulator consists of four sequentially connected glass vessels, V1 to $\mathrm{V} 4$, to model different sections of the human colon from the most proximal to distal area. Vessels V1, V2, V3, and V4 represent the ascending, transverse, descending and sigmoid colon, respectively. A synthetic ileal simulation medium (containing starch, peptone, tryptone, yeast extract, $\mathrm{NaCl}, \mathrm{KCl}$, mucin, casein, pectin, xylan, arabinogalactan, $\mathrm{NaHCO}_{3}$, $\mathrm{MgSO}_{4}$, guar gum, inulin, cysteine, $\mathrm{KH}_{2} \mathrm{PO}_{4}, \mathrm{~K}_{2} \mathrm{HPO}_{4}$, bile salts, $\mathrm{CaCl}_{2}, \mathrm{FeSO}_{4}$, hemin and tween 80 ) was prepared as previously described [21] and was used as the simulation control (SC). The test substrates polydextrose (PDX), lactitol, and xylitol were obtained from Danisco Sweeteners Oy, Kantvik, Finland. For the simulations, 2\% mixtures of the test substrates were prepared in the simulation medium. Two separate simulations were run for $48 \mathrm{~h}$ whereafter the fecal simulation slurries were collected from all vessels. All samples were stored at $-80^{\circ} \mathrm{C}$ prior to analysis.

\subsection{Analysis of Short Chain Fatty Acids and Lactic Acid}

The short-chain fatty acids and lactic acid in fecal simulation slurries were analyzed using the method by Ouwehand et al. with modifications [26]. In short, internal standard (100 $\mu \mathrm{L} 20 \mathrm{mM}$ pivalic acid), $300 \mu \mathrm{L}$ of water and $250 \mu \mathrm{L}$ of saturated oxalic acid solution were added to $100 \mu \mathrm{L}$ of the sample. After thorough mixing, the sample was incubated at $4{ }^{\circ} \mathrm{C}$ for $60 \mathrm{~min}$, and then centrifuged at $16,000 \times \mathrm{g}$ for $5 \mathrm{~min}$. The supernatant was analyzed by gas chromatography using a glass column packed with 80/120 Carbopack 
B-DA/4\% Carbowax $20 \mathrm{M}$ stationary phase $(2 \mathrm{~m} \times 2 \mathrm{~mm}$, Supelco, Bellefonte, PA, USA) at $175{ }^{\circ} \mathrm{C}$ using helium as the carrier gas at flow rate of $24 \mathrm{~mL} / \mathrm{min}$. The temperature of the injector and the flame ionization detector were $200{ }^{\circ} \mathrm{C}$ and $245{ }^{\circ} \mathrm{C}$, respectively. The concentration of acetic acid, propionic acid, butyric acid, iso-butyric acid, valeric acid, isovaleric acid, 2-methylbutyric acid and lactic acid was determined.

\subsection{HT29-MTX-E12 Cell Culture and Treatments}

The human mucus-secreting colonic cell line HT29-MTX-E12 was a kind gift from Dr. David Brayden and Dr. Sam Mayer (UCD Conway Institute, Dublin, Ireland). E12 cells were maintained in growth medium consisting of Dulbecco's Modified Eagle medium (DMEM, Gibco, Thermo Fisher Scientific, Waltham, MA, USA) supplemented with 10\% fetal calf serum (FCS, Bio-Whittaker, Lonza, Belgium), 4 mM GlutaMax (Gibco), $10 \mathrm{mM}$ HEPES buffer solution (Gibco), $100 \mathrm{U} / \mathrm{mL}$ penicillin and $100 \mu \mathrm{g} / \mathrm{mL}$ streptomycin (SigmaAldrich, St. Louis, MO, USA) [23]. Before the experiments, cells were seeded at a density of $2 \times 10^{5}$ cells $/ \mathrm{cm}^{2}$ on polyethylene terephthalate cell culture inserts with a pore size of $0.4 \mu \mathrm{m}$ in 6-well plates (BD Bioscience, Franklin Lakes, NJ, USA), and cultured in 5\% $\mathrm{CO}_{2}$ humidified atmosphere at $37^{\circ} \mathrm{C}$ for 43 days until fully differentiated as determined by transepithelial electrical resistance (TEER). Deoxynivalenol (DON, Sigma-Aldrich) was dissolved in 96\% ethanol and further diluted in growth medium without FCS. E12 cells were then exposed to $50 \mu \mathrm{M}$ DON for 3 days, after which the DON was removed and compromised cell monolayer was treated with $5 \%$ fermentation products in growth medium without FCS for another 5 days (until d 51). Growth medium without FCS and fermentation products was used as negative control, and growth medium with $10 \%$ FCS was used as positive control. All cell assays were conducted twice for each batch of fermentation product. All solutions were filter-sterilized.

\subsection{Evaluation of Epithelial Barrier Function}

The epithelial barrier function is evaluated by measuring TEER and paracellular permeability at $\mathrm{d} 51$ as previous described [23]. TEER was measured in triplicate wells using a Millicell-ERS Volt-ohmmeter (Millipore, Billerica, MA, USA) according to the manufacturer's instructions. Paracellular permeability was determined by measuring the flux of $4 \mathrm{kD}$ fluorescein isothiocyanate-dextran (FITC-D4, Sigma-Aldrich) across the E12 cell monolayer. Briefly, FITC-D4 was added to the apical compartment of the cell inserts at a final concentration of $2.2 \mathrm{mg} / \mathrm{mL}$ in FCS-free growth medium. After $1 \mathrm{~h}$ incubation at $37^{\circ} \mathrm{C}$, FITC-D4 in each basolateral compartment was measured in duplicates using an EnVision 2103 Multilabel Reader (PerkinElmer, Waltham, MA, USA). Wavelengths of excitation and emission were set at $485 \mathrm{~nm}$ and $538 \mathrm{~nm}$, respectively. Data were presented as mean \pm SEM, relative to the negative control (without fermentation products and FCS) in each cell assay.

\subsection{Statistical Analysis}

The in vitro colonic fermentation was conducted twice, and cell assays were also performed twice with duplicates for each fermentation. Data was analyzed using statistical software R version 3.6.1 (R Core Team, Vienna, Austria) [27]. Data on SCFA production, TEER and permeability was analyzed by two-way ANOVA in a mixed model using substrates, simulation vessels (V1 to V4) and their interactions as fixed effects, whereas simulation and assay numbers were considered as random effects. Normality of data was tested by Shapiro-Wilk normality test and all data are normally distributed. Differences among treatments were analyzed by Tukey's test. Simple correlations between cellular parameters (TEER and permeability) and SCFAs were evaluated by Pearson's correlation analysis. A $p<0.05$ was considered significant. 


\section{Results}

\subsection{SCFAs Production during Simulated Colonic Fermentation}

After the in vitro colonic fermentation, the composition of SCFAs was analyzed. SCFAs were produced due to the three substrates, but a small amount of SCFAs was also produced throughout the four vessels due to the carbohydrate components such as starch, inulin, guar gum etc. contained in the simulation medium. Overall, total SCFA production was between 43 to $176 \mathrm{mM}$, with some variation among different substrates (Figure 1). The fermentation of simulation control (SC), PDX, and lactitol produced mainly acetate, while xylitol fermentation produced mainly butyrate, and this pattern was constant throughout V1-V4.

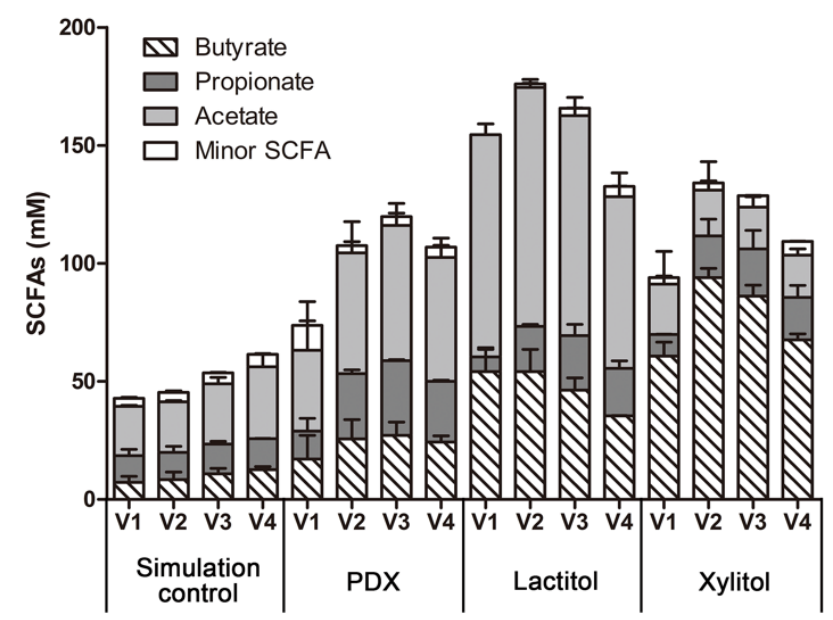

Figure 1. Overall SCFA production profile during in vitro fermentation. Polydextrose (PDX), lactitol and xylitol was fermented in vitro by the EnteroMix fermentation model, simulation medium was used as simulation control (SC) $(\mathrm{n}=2)$. The production of acetate, propionate, butyrate and minor SCFAs (iso-butyrate, 2-methylbutyrate, isovalerate, valerate, lactate) was measured. V1-V4, vessel 1 to vessel 4, represent the ascending, transverse, descending and sigmoid colon. Data are presented as mean \pm SEM (mM). For detailed statistical analysis, see Figure 2.
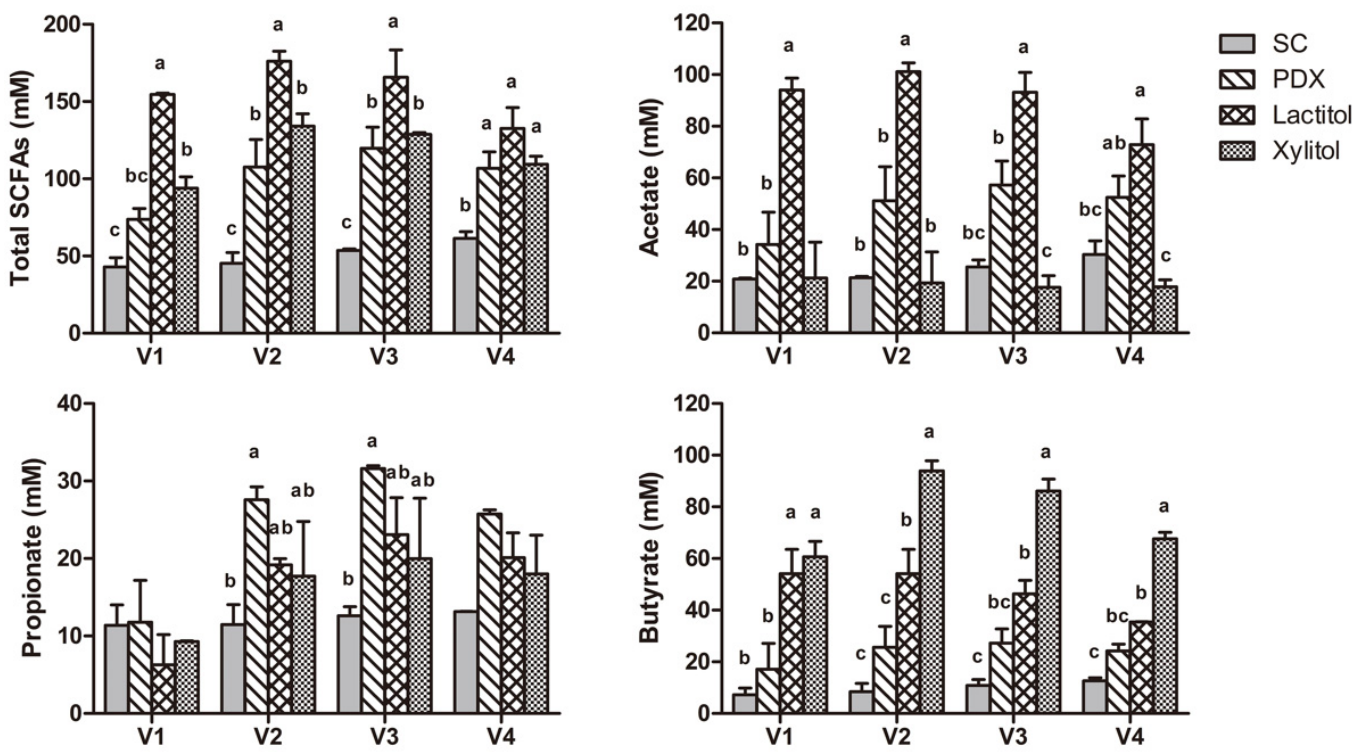

Figure 2. Production of total and major SCFAs during in vitro fermentation. Polydextrose (PDX), lactitol and xylitol was fermented in vitro by the EnteroMix fermentation model, and simulation medium was used as simulation control (SC). V1-V4, vessel 1 to vessel 4, represent the ascending, transverse, descending and sigmoid colon. The production of acetate, propionate, butyrate and total SCFAs is presented as mean \pm SEM of two fermentations $(n=2)$. Within each vessel (V1-V4), bars with different letters differ significantly at $p<0.05$ according to Tukey's test. 
When compared with SC, all three substrates increased total SCFA production significantly except PDX in V1 ( $p=0.106$, Figure 2$)$. The total SCFAs production was tripled by lactitol fermentation $(\sim 176 \mathrm{mM})$ and doubled by PDX and xylitol fermentation $(\sim 134 \mathrm{mM})$ from V1 to V3, but it was not significantly different between the 3 substrates in V4 (133 mM, $107 \mathrm{mM}$, and $109 \mathrm{mM}$, respectively). For individual SCFAs, although PDX fermentation doubled acetate and butyrate concentrations compared with SC, they were not statistically different. Lactitol fermentation resulted in the highest acetate production $(\sim 101 \mathrm{mM}$, $p<0.05)$ in V1-3 among all simulations. Xylitol fermentation did not increase acetate concentration as compared with SC, but resulted in the highest butyrate production $(\sim 94 \mathrm{mM}$, $p<0.01$ ) except V1. Propionate production was almost constant among substrates and vessels, but only PDX fermentation led to a small increase in V2 and V3 compared with SC $(\sim 32 \mathrm{mM}, p<0.05)$.

Despite the differences in actual concentrations, the proportion of each individual SCFA during the fermentation of SC, PDX, and lactitol did not differ. Xylitol fermentation resulted in significantly lower acetate percent except V1 $(14-22 \%, p<0.01)$ and higher butyrate percent $(62-70 \%, p<0.01)$ due to the production of butyrate (Table 1$)$.

Table 1. Major short chain fatty acids production shown by proportion during in vitro fermentation.

\begin{tabular}{|c|c|c|c|c|c|}
\hline & \multirow{2}{*}{ Vessels } & \multicolumn{4}{|c|}{ Substrates } \\
\hline & & SC & PDX & Lactitol & Xylitol \\
\hline \multirow[t]{4}{*}{ Acetate } & V1 & $49.6 \pm 6.1 \%{ }^{a}$ & $45.2 \pm 12.6 \% \mathrm{ab}$ & $60.9 \pm 3.3 \% \mathrm{a}$ & $21.6 \pm 13.1 \% b$ \\
\hline & $\mathrm{V} 2$ & $48.1 \pm 6.2 \%^{\mathrm{a}}$ & $46.9 \pm 4.5 \%{ }^{\mathrm{a}}$ & $57.6 \pm 4.0 \%{ }^{\mathrm{a}}$ & $14.0 \pm 8.1 \% \mathrm{~b}$ \\
\hline & V3 & $47.8 \pm 5.9 \%^{\mathrm{a}}$ & $47.6 \pm 2.3 \%{ }^{a}$ & $56.3 \pm 1.3 \%{ }^{a}$ & $13.7 \pm 3.4 \% \mathrm{~b}$ \\
\hline & V4 & $49.0 \pm 5.1 \%^{\mathrm{a}}$ & $48.9 \pm 2.9 \%{ }^{\mathrm{a}}$ & $54.7 \pm 2.0 \% \mathrm{a}$ & $16.2 \pm 1.7 \% \mathrm{~b}$ \\
\hline \multirow[t]{4}{*}{ Propionate } & V1 & $26.2 \pm 2.4 \%^{\mathrm{a}}$ & $16.8 \pm 8.9 \%$ ab & $4.1 \pm 2.6 \% \mathrm{~b}$ & $9.9 \pm 0.7 \% \mathrm{~b}$ \\
\hline & $\mathrm{V} 2$ & $25.0 \pm 1.9 \% \mathrm{ab}$ & $26.6 \pm 6.0 \%{ }^{a}$ & $10.9 \pm 0.0 \% \mathrm{~b}$ & $13.6 \pm 6.1 \% \mathrm{ab}$ \\
\hline & V3 & $23.5 \pm 1.7 \%$ & $26.7 \pm 2.7 \%$ & $13.8 \pm 1.4 \%$ & $15.6 \pm 6.2 \%$ \\
\hline & V4 & $21.5 \pm 1.6 \%$ & $24.3 \pm 1.9 \%$ & $15.1 \pm 0.9 \%$ & $16.3 \pm 3.8 \%$ \\
\hline \multirow[t]{4}{*}{ Butyrate } & V1 & $16.3 \pm 3.8 \% \mathrm{~b}$ & $22.2 \pm 11.4 \% \mathrm{~b}$ & $35.0 \pm 5.9 \% \mathrm{~b}$ & $65.5 \pm 11.5 \%{ }^{a}$ \\
\hline & V2 & $18.0 \pm 4.2 \% \mathrm{~b}$ & $23.3 \pm 3.7 \% \mathrm{~b}$ & $30.6 \pm 4.2 \% \mathrm{~b}$ & $70.2 \pm 1.3 \%{ }^{a}$ \\
\hline & V3 & $20.1 \pm 4.0 \% \mathrm{~b}$ & $22.5 \pm 2.1 \% \mathrm{~b}$ & $28.0 \pm 0.2 \% \mathrm{~b}$ & $66.9 \pm 3.0 \%{ }^{a}$ \\
\hline & $\mathrm{V} 4$ & $20.9 \pm 3.5 \% \mathrm{~b}$ & $22.7 \pm 0.2 \% \mathrm{~b}$ & $27.0 \pm 2.7 \% b$ & $62.1 \pm 5.3 \% \mathrm{a}$ \\
\hline
\end{tabular}

Data are presented as mean percent \pm SEM of two fermentations $(n=2)$. Within a row, numbers with different letters differ significantly at $p<0.05$ according to Tukey's test. SC, simulation control; PDX, polydextrose.

\subsection{Effect of Fermentation Products on Epithelial Barrier Function}

When compared with the negative control (growth medium without FCS and fermentation products), all fermentation products including that of SC alone significantly increased TEER $(123-168 \%, p<0.001)$ and decreased the paracellular permeability (36-64\%, $p<0.001$ ) of compromised E12 monolayers (Figure 3). The effects of PDX fermentation products on TEER and permeability did not differ from that of SC. Lactitol fermentation products increased TEER to an equivalent level compared with that of SC, but those from $\mathrm{V} 2$ and V4 resulted in lower permeability $(p<0.01)$. Xylitol fermentation products from V3 and V4 significantly increased TEER $(p<0.001)$ and decreased permeability $(p<0.01)$ compared with SC, and fermentation products from V4 led to highest TEER $(p<0.001)$ while that from V3 resulted in the lowest permeability $(p<0.05)$. 

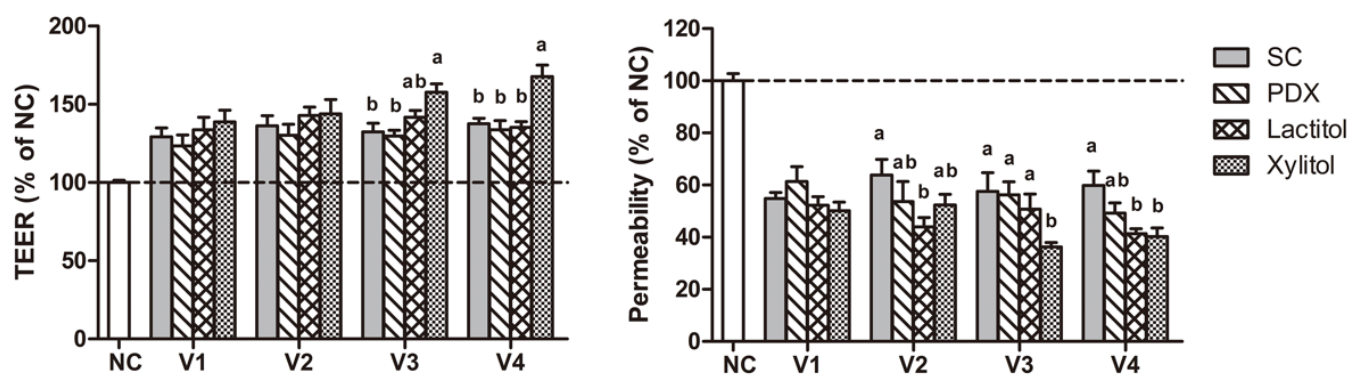

Figure 3. Effect of fermentation products on the epithelial barrier function. HT29-MTX-E12 cells were pre-treated with deoxynivalenol, and then $5 \%$ fermentation products of simulation control (SC), polydextrose (PDX), lactitol and xylitol were added. Transepithelial electrical resistance (TEER) and paracellular permeability data were obtained from four independent cell assays with duplicates $(\mathrm{n}=8)$, and presented as mean values $\pm \mathrm{SEM}$, relative to negative control $(\mathrm{NC}=100 \%)$. NC indicates growth medium without FCS and fermentation products. V1-V4 indicates vessel 1 to vessel 4 in in vitro EnteroMix fermentation model. All fermentation products differed significantly with NC $(p<0.001)$, and only differences between fermentation products are shown in the figure. Different lowercase letters indicate significant differences between four fermentation products within each vessel at $p<0.05$ according to Tukey's test.

To better understand the relation between SCFAs and epithelial barrier function, correlation analysis was carried out (Table 2). Total SCFAs and butyrate concentration, as well as butyrate proportion, were positively correlated to TEER $(p<0.05)$, and negatively correlated to permeability $(p<0.001)$. However, acetate and propionate concentration were not correlated to TEER or permeability, but their proportion were negatively correlated to TEER $(p<0.05)$ and positively correlated to permeability $(p<0.05)$.

Table 2. Correlations between cellular parameters (TEER and permeability) and SCFAs.

\begin{tabular}{ccccc}
\hline & \multicolumn{2}{c}{ TEER } & \multicolumn{2}{c}{ Permeability } \\
\cline { 2 - 5 } & $\boldsymbol{r}$ & $\boldsymbol{p}$ & $\boldsymbol{r}$ & $\boldsymbol{p}$ \\
\hline Actual concentration (mM) & & & -0.106 & 0.249 \\
Acetate & -0.096 & 0.293 & -0.109 & 0.234 \\
Propionate & 0.055 & 0.543 & -0.391 & $<0.001$ \\
Butyrate & 0.428 & $<0.001$ & -0.334 & $<0.001$ \\
Total SCFAs & 0.204 & 0.024 & & \\
Proportion of total SCFAs & & & 0.221 & 0.015 \\
Acetate & -0.333 & $<0.001$ & 0.231 & 0.011 \\
Propionate & -0.212 & 0.019 & -0.368 & $<0.001$ \\
Butyrate & 0.450 & $<0.001$ &
\end{tabular}

$r$, Pearson's correlation coefficient. $p$, significant level, $p<0.05$ was considered significant.

\section{Discussion}

In recent decades, PDX, lactitol and xylitol have increasingly been investigated in gastrointestinal research, especially in those studies related to colon health. Their beneficial effects on colon epithelium are mostly achieved by the modification of microbiota and the production of SCFAs [16-18]. In concert with a previous study, xylitol fermentation mainly leads to a dramatic increase of butyrate [20]. In our study, PDX fermentation increased all three major SCFAs to a small extent, but not as much as lactitol and xylitol and not statistically differently to the control. Interestingly, although there are small increases in actual amounts, the proportion of individual SCFAs remained constant throughout V1 to $\mathrm{V} 4$, and the fermentation pattern was the same as reported in other studies [20,21]. The reason that PDX fermentation only led to small insignificant increases may be attributed to its slow fermentation [28]. Because of its complex structure, PDX is gradually degraded and fermented by the colonic microbiota, and therefore leads to a sustained production of SCFAs from proximal to distal colon [17]. Our results also showed that lactitol fermentation increased acetate the most, followed by butyrate, which is consistent with the findings of 
Makivuokko et al. [19]. However, Probert et al. reported that the main SCFA in lactitol fermentation products is butyrate [29], whereas Beards et al. showed that acetate and propionate increased by the same amount [30]. This inconsistency may be due to the difference in the in vitro fermentation methods.

Few previous studies have reported that SCFA mixtures and in vitro fermentation products can improve intestinal barrier function $[23,31]$. Our results also showed that all fermentation products increased TEER and decreased paracellular permeability of the compromised E12 cell monolayer, indicating that the production of SCFAs promotes intestinal barrier repair. Notably, despite the huge variation in actual SCFA concentrations, the fermentation products of PDX and lactitol did not provide additional benefits compared with SC. One possible reason could be that only $5 \%$ of the fermentation products were added to the cell culture medium, thus the variation in final SCFA concentration may not be large enough to make a difference to barrier repair. Nevertheless, xylitol fermentation products showed better barrier repairing effect at the same low concentration level, suggesting the small difference in final SCFA concentrations may not be the main reason for the lack of effect. In our study, fermentation of SC, PDX, and lactitol produced mainly acetate, resulting in a constant SCFA proportion, while xylitol fermentation produced mainly butyrate, and led to much higher butyrate proportion. It seems that the better barrier repairing effects of xylitol fermentation products were attributed to butyrate, as also supported by the correlation analysis. Although all three major SCFAs were reported to improve intestinal barrier function, butyrate was likely to be the most potent $[7,8,13]$. Butyrate is mostly utilized locally by colonocytes as a direct energy source, and provides $60-70 \%$ of the energy that is required for colonic cell proliferation and differentiation [14]. Conversely, acetate and propionate act more systemically in vivo, as the majority are rapidly absorbed and transported to the liver, and used as substrates for gluconeogenesis, lipogenesis, protein synthesis or cholesterol synthesis [3,5]. Moreover, the discrepancy among acetate, propionate and butyrate was also assumed as partially related to their different ability to cross the cell membrane [7]. Nevertheless, in a previous study with the same cell model, no consistency between the cellular effects (including barrier function and gene expression) and the levels of butyrate in the fermentation products was observed [23]. Yet, correlation analysis was not conducted, and the concentration of acetate and propionate were not measured in that study. The missing information makes direct comparison with our study very difficult, as the beneficial effects on barrier function may also come from high butyrate proportion [31]. Chen et al. found that SCFA mixtures with $20 \%$ and $50 \%$ butyrate repaired disrupted barrier function, and 50\% butyrate further increased the TEER value [31]. In our study, fermentation products with 16-35\% butyrate (SC, PDX and lactitol) improved barrier function to an equivalent level, while fermentation products with 60-70\% butyrate (xylitol) led to a better barrier repair. Our observation fits well with the findings of Chen et al., suggesting that the proportion of butyrate may actually also be a key factor for barrier repair. Hence, the better reparative effects of xylitol fermentation products observed in our study are likely to be due to the high production of butyrate. It has been reported that butyrate facilitates cell differentiation, and enhances intestinal barrier function by reinforcing the tight junctions $[3,32,33]$, as well as increasing mucin production [3,34]. Our results imply that in colonic fermentation products, butyrate may play a major role in intestinal barrier repair. Of course, the benefits of acetate and propionate cannot be excluded $[10,11,35]$; there may even be synergistic effects with butyrate.

Our results showed that xylitol may be more potent than PDX and lactitol in improving intestinal barrier function. However, it was found that low concentrations of SCFAs promote intestinal barrier function, while high concentrations have no beneficial effects and may even disrupt barrier function $[23,31]$. Thus, fibers with slow but steady SCFA production like PDX could be beneficial in vivo compared to quickly fermented ones that cause localized high SCFA concentrations $[17,31]$. In contrast, xylitol is readily fermented by colonic microbiota, but it is important to note that half of the consumed xylitol is absorbed in the small intestine in vivo, which leaves only $50 \%$ of the dietary xylitol available 
for colonic fermentation [18]. Therefore, their effects in vivo may not be the same as shown in vitro, and its promising effects warrant further studies. In addition, acetate is a fermentation product of most gut bacteria, whereas butyrate and propionate are produced by more specific bacterial species [3]. Although all these ingredients have shown prebiotic potential $[17,18,36]$, apparently, they have different fermentation patterns, which could be influenced by different microbial composition. Unfortunately, the changes in the microbiome were not assessed in the present study, but it would be worthy investigating how these changes could influence the observed results.

\section{Conclusions}

In conclusion, the current study combined an in vitro EnteroMix semi-continuous colonic fermentation simulator and a mucus-secreting human HT29-MTX-E12 cell model, simulated the fermentation of three widely used food ingredients and their effects on impaired colonic epithelial barrier function in vivo. Our results showed that fermentation of PDX, lactitol and xylitol produced different amount of SCFAs, and their fermentation products promoted the repair of disrupted intestinal barrier in vitro. Among the three tested ingredients, xylitol fermentation resulted in better reparative effects than PDX and lactitol, and this is likely to be due to the high production of butyrate. Therefore, with regard to the selection of functional ingredients for improving gut health related to chronic diseases, the ingredients that mainly produce butyrate during fermentation may of particularly interests.

Author Contributions: Conceptualization, K.E.B.K., M.T.S., A.C.O. and S.P.; Formal analysis, Y.Y.; Funding acquisition, K.E.B.K.; Investigation, D.S.G.N., S.D.F., M.T.S. and S.P.; Project administration, M.T.S. and S.P.; Resources, K.E.B.K., M.T.S., A.C.O. and S.P.; Supervision, K.E.B.K., M.T.S., A.C.O. and S.P.; Validation, D.S.G.N., S.D.F., M.T.S. and S.P.; Visualization, Y.Y.; Writing-Original draft, Y.Y.; Writing - Review \& Editing, Y.Y. All authors have read and agreed to the published version of the manuscript.

Funding: This research was funded by the Danish Strategic Research Fund, grant number 10-93526.

Data Availability Statement: Data is contained within the article.

Acknowledgments: The authors wish to thank Annette K. Nielsen, Kasper V. Poulsen for their excellent technical assistance.

Conflicts of Interest: The authors declare no conflict of interest.

\section{References}

1. Viggiano, D.; Ianiro, G.; Vanella, G.; Bibbo, S.; Bruno, G.; Simeone, G.; Mele, G. Gut barrier in health and disease: Focus on childhood. Eur. Rev. Med. Pharmacol. Sci. 2015, 19, 1077-1085. [PubMed]

2. Vaziri, N.D.; Zhao, Y.Y.; Pahl, M.V. Altered intestinal microbial flora and impaired epithelial barrier structure and function in CKD: The nature, mechanisms, consequences and potential treatment. Nephrol. Dial. Transpl. 2016, 31, 737-746. [CrossRef]

3. Martin-Gallausiaux, C.; Marinelli, L.; Blottiere, H.M.; Larraufie, P.; Lapaque, N. SCFA: Mechanisms and functional importance in the gut. Proc. Nutr. Soc. 2021, 80, 37-49. [CrossRef] [PubMed]

4. Parada Venegas, D.; De la Fuente, M.K.; Landskron, G.; Gonzalez, M.J.; Quera, R.; Dijkstra, G.; Harmsen, H.J.M.; Faber, K.N.; Hermoso, M.A. Short Chain Fatty Acids (SCFAs)-Mediated Gut Epithelial and Immune Regulation and Its Relevance for Inflammatory Bowel Diseases. Front. Immunol. 2019, 10, 277. [CrossRef] [PubMed]

5. van der Beek, C.M.; Dejong, C.H.C.; Troost, F.J.; Masclee, A.A.M.; Lenaerts, K. Role of short-chain fatty acids in colonic inflammation, carcinogenesis, and mucosal protection and healing. Nutr. Rev. 2017, 75, 286-305. [CrossRef]

6. $\quad$ Bach Knudsen, K.E.; Laerke, H.N.; Hedemann, M.S.; Nielsen, T.S.; Ingerslev, A.K.; Gundelund Nielsen, D.S.; Theil, P.K.; Purup, S.; Hald, S.; Schioldan, A.G.; et al. Impact of Diet-Modulated Butyrate Production on Intestinal Barrier Function and Inflammation. Nutrients 2018, 10, 1499. [CrossRef]

7. Elamin, E.E.; Masclee, A.A.; Dekker, J.; Pieters, H.J.; Jonkers, D.M. Short-chain fatty acids activate AMP-activated protein kinase and ameliorate ethanol-induced intestinal barrier dysfunction in Caco-2 cell monolayers. J. Nutr. 2013, 143, 1872-1881. [CrossRef]

8. D'Souza, W.N.; Douangpanya, J.; Mu, S.; Jaeckel, P.; Zhang, M.; Maxwell, J.R.; Rottman, J.B.; Labitzke, K.; Willee, A.; Beckmann, H.; et al. Differing roles for short chain fatty acids and GPR43 agonism in the regulation of intestinal barrier function and immune responses. PLoS ONE 2017, 12, e0180190. [CrossRef] 
9. Suzuki, T.; Yoshida, S.; Hara, H. Physiological concentrations of short-chain fatty acids immediately suppress colonic epithelial permeability. Br. J. Nutr. 2008, 100, 297-305. [CrossRef]

10. Xia, Z.; Han, Y.; Wang, K.; Guo, S.; Wu, D.; Huang, X.; Li, Z.; Zhu, L. Oral administration of propionic acid during lactation enhances the colonic barrier function. Lipids Health Dis. 2017, 16, 62. [CrossRef]

11. Tong, L.C.; Wang, Y.; Wang, Z.B.; Liu, W.Y.; Sun, S.; Li, L.; Su, D.F.; Zhang, L.C. Propionate Ameliorates Dextran Sodium SulfateInduced Colitis by Improving Intestinal Barrier Function and Reducing Inflammation and Oxidative Stress. Front. Pharmacol. 2016, 7, 253. [CrossRef]

12. Chen, G.; Ran, X.; Li, B.; Li, Y.; He, D.; Huang, B.; Fu, S.; Liu, J.; Wang, W. Sodium Butyrate Inhibits Inflammation and Maintains Epithelium Barrier Integrity in a TNBS-induced Inflammatory Bowel Disease Mice Model. EBioMedicine 2018, 30, 317-325. [CrossRef]

13. Ohata, A.; Usami, M.; Miyoshi, M. Short-chain fatty acids alter tight junction permeability in intestinal monolayer cells via lipoxygenase activation. Nutrition 2005, 21, 838-847. [CrossRef] [PubMed]

14. Tan, J.; McKenzie, C.; Potamitis, M.; Thorburn, A.N.; Mackay, C.R.; Macia, L. The role of short-chain fatty acids in health and disease. Adv. Immunol. 2014, 121, 91-119. [CrossRef] [PubMed]

15. Morrison, D.J.; Preston, T. Formation of short chain fatty acids by the gut microbiota and their impact on human metabolism. Gut Microbes 2016, 7, 189-200. [CrossRef] [PubMed]

16. Drakoularakou, A.; Hasselwander, O.; Edinburgh, M.; Ouwehand, A. Lactitol, an emerging prebiotic: Functional properties with a focus on digestive health. Food Sci. Technol. Bull. Funct. Foods 2007, 3, 71-80. [CrossRef]

17. do Carmo, M.M.; Walker, J.C.; Novello, D.; Caselato, V.M.; Sgarbieri, V.C.; Ouwehand, A.C.; Andreollo, N.A.; Hiane, P.A.; Dos Santos, E.F. Polydextrose: Physiological Function, and Effects on Health. Nutrients 2016, 8, 553. [CrossRef]

18. Salli, K.; Lehtinen, M.J.; Tiihonen, K.; Ouwehand, A.C. Xylitol's Health Benefits beyond Dental Health: A Comprehensive Review. Nutrients 2019, 11, 1813. [CrossRef]

19. Makivuokko, H.; Forssten, S.; Saarinen, M.; Ouwehand, A.; Rautonen, N. Synbiotic effects of lactitol and Lactobacillus acidophilus NCFM in a semi-continuous colon fermentation model. Benef. Microbes 2010, 1, 131-137. [CrossRef]

20. Makelainen, H.S.; Makivuokko, H.A.; Salminen, S.J.; Rautonen, N.E.; Ouwehand, A.C. The effects of polydextrose and xylitol on microbial community and activity in a 4-stage colon simulator. J. Food Sci. 2007, 72, M153-M159. [CrossRef] [PubMed]

21. Makivuokko, H.; Nurmi, J.; Nurminen, P.; Stowell, J.; Rautonen, N. In vitro effects on polydextrose by colonic bacteria and caco-2 cell cyclooxygenase gene expression. Nutr. Cancer 2005, 52, 94-104. [CrossRef]

22. Behrens, I.; Stenberg, P.; Artursson, P.; Kissel, T. Transport of lipophilic drug molecules in a new mucus-secreting cell culture model based on HT29-MTX cells. Pharm. Res. 2001, 18, 1138-1145. [CrossRef]

23. Nielsen, D.S.G.; Jensen, B.B.; Theil, P.K.; Nielsen, T.S.; Knudsen, K.E.B.; Purup, S. Effect of butyrate and fermentation products on epithelial integrity in a mucus-secreting human colon cell line. J. Funct. Foods 2018, 40, 9-17. [CrossRef]

24. Lamichhane, S.; Yde, C.C.; Jensen, H.M.; Morovic, W.; Hibberd, A.A.; Ouwehand, A.C.; Saarinen, M.T.; Forssten, S.D.; Wiebe, L.; Marcussen, J.; et al. Metabolic Fate of (13)C-Labeled Polydextrose and Impact on the Gut Microbiome: A Triple-Phase Study in a Colon Simulator. J. Proteome Res. 2018, 17, 1041-1053. [CrossRef]

25. Forssten, S.; Ouwehand, A.C. Dose-Response Recovery of Probiotic Strains in Simulated Gastro-Intestinal Passage. Microorganisms 2020, 8, 112. [CrossRef] [PubMed]

26. Ouwehand, A.C.; Tiihonen, K.; Saarinen, M.; Putaala, H.; Rautonen, N. Influence of a combination of Lactobacillus acidophilus NCFM and lactitol on healthy elderly: Intestinal and immune parameters. Br. J. Nutr. 2009, 101, 367-375. [CrossRef]

27. Team, R.C. R: A Language and Environment for Statistical Computing; R Foundation for Statistical Computing: Vienna, Austria, 2019.

28. Roytio, H.; Ouwehand, A.C. The fermentation of polydextrose in the large intestine and its beneficial effects. Benef. Microbes 2014, 5, 305-313. [CrossRef] [PubMed]

29. Probert, H.M.; Apajalahti, J.H.; Rautonen, N.; Stowell, J.; Gibson, G.R. Polydextrose, lactitol, and fructo-oligosaccharide fermentation by colonic bacteria in a three-stage continuous culture system. Appl. Environ. Microbiol. 2004, 70, 4505-4511. [CrossRef]

30. Beards, E.; Tuohy, K.; Gibson, G. Bacterial, SCFA and gas profiles of a range of food ingredients following in vitro fermentation by human colonic microbiota. Anaerobe 2010, 16, 420-425. [CrossRef] [PubMed]

31. Chen, T.; Kim, C.Y.; Kaur, A.; Lamothe, L.; Shaikh, M.; Keshavarzian, A.; Hamaker, B.R. Dietary fibre-based SCFA mixtures promote both protection and repair of intestinal epithelial barrier function in a Caco-2 cell model. Food Funct. 2017, 8, 1166-1173. [CrossRef] [PubMed]

32. Zheng, L.; Kelly, C.J.; Battista, K.D.; Schaefer, R.; Lanis, J.M.; Alexeev, E.E.; Wang, R.X.; Onyiah, J.C.; Kominsky, D.J.; Colgan, S.P. Microbial-Derived Butyrate Promotes Epithelial Barrier Function through IL-10 Receptor-Dependent Repression of Claudin-2. J. Immunol. 2017, 199, 2976-2984. [CrossRef]

33. Wang, H.B.; Wang, P.Y.; Wang, X.; Wan, Y.L.; Liu, Y.C. Butyrate enhances intestinal epithelial barrier function via up-regulation of tight junction protein Claudin-1 transcription. Dig. Dis. Sci. 2012, 57, 3126-3135. [CrossRef] [PubMed]

34. Gaudier, E.; Jarry, A.; Blottiere, H.M.; de Coppet, P.; Buisine, M.P.; Aubert, J.P.; Laboisse, C.; Cherbut, C.; Hoebler, C. Butyrate specifically modulates MUC gene expression in intestinal epithelial goblet cells deprived of glucose. Am. J. Physiol. Gastrointest. Liver Physiol. 2004, 287, G1168-G1174. [CrossRef] 
35. Wan Saudi, W.S.; Sjoblom, M. Short-chain fatty acids augment rat duodenal mucosal barrier function. Exp. Physiol. 2017, 102, 791-803. [CrossRef] [PubMed]

36. Li, X.Q.; Zhang, X.M.; Wu, X.; Lan, Y.; Xu, L.; Meng, X.C.; Li, J.N. Beneficial effects of lactitol on the composition of gut microbiota in constipated patients. J. Dig. Dis. 2020, 21, 445-453. [CrossRef] [PubMed] 